Amazonía Peruana, Volumen XVII, №34, 2021; pp. 279-286

\title{
Obituario, Gerald Weiss (1932-2021)
}

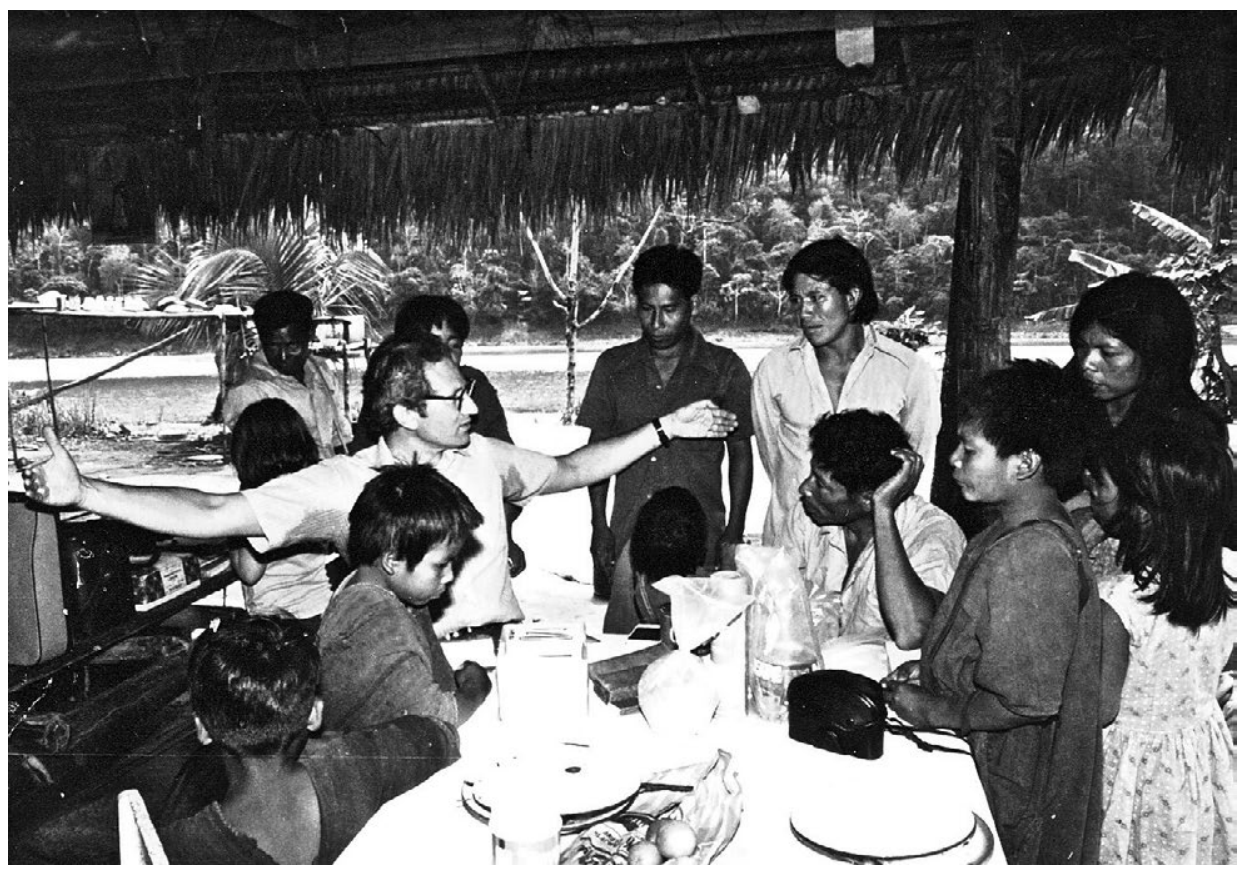

Figura 1: Gerald Weiss trabajando en Otika, a fines de los 1970 (cortesía de Eric Weiss). 
E1 Dr. Gerald Weiss, profesor emérito de Antropología de la Florida Atlantic University (Estados Unidos) — cuyo estudio perspicaz, sensible y meticuloso de la cultura asháninka ayudó a sentar las bases empíricas y teóricas para el trabajo etnográfico en la Selva Central—falleció en Boca Ratón, Florida, el 10 de abril de 2021, a los 88 años. Gerald Weiss nació en el Bronx, Nueva York el 29 de junio de 1932; hijo de Max Weiss, nativo de Iași, Rumania, y Rose Knopping. Asistió a la Escuela Secundaria de Ciencias del Bronx y a la Universidad de Columbia.

En 1952, Weiss comenzó sus estudios de antropología en la Universidad de Míchigan, interrumpidos por dos años de servicio en el ejército de los Estados Unidos en 1954. Recibió una maestría, y aprobó sus exámenes de calificación en 1958, después de lo cual regresó a Nueva York. Como dijo en una carta a un miembro anónimo del profesorado de la Universidad de Michigan en septiembre de 1959, "Me enfrenté al problema de seleccionar una cultura para el estudio de campo. Mi dificultad radicaba en mi total falta de preferencia por cualquier parte en particular del mundo". En consulta, en parte, con Robert Carneiro (1927-2020), antropólogo del Museo Americano de Historia Natural, Weiss abandonó un proyecto naciente sobre sistemas políticos africanos comparativos para realizar investigaciones entre el pueblo asháninka del Perú.

Con el apoyo financiero de la Fundación Nacional de Ciencias y el Consejo de Investigación en Ciencias Sociales, Weiss llegó a Lima a fines de septiembre de 1960 a bordo del barco Santa Margarita, pasando seis semanas en lo que describió en su diario como el "lugar de reunión del antropólogo", Pensión Morris en Breña. También estuvieron personajes como el antropólogo Michael Harner (1929-2018) - ya un etnógrafo experimentado en los pueblos jíbaro y luego afiliado al Museo Americano de Historia Natural — que estaba a punto de emprender un viaje a las comunidades konibo. Por consejo de Willard Kindberg, del Instituto Lingüístico de Verano (ILV), en noviembre, Weiss, con sus 700 kilos de equipo, viajó por tierra a San Ramón, donde pasó unas dos semanas, antes de llegar en avión, a principios de diciembre, a Puerto Ocopa. En sus intentos posteriores de asegurar un bote, motor y otras provisiones, Weiss no se instalaría en una comunidad asháninka durante otros seis meses. Al llegar a Atalaya en balsa a mediados de diciembre, pero sin poder comprar las provisiones pertinentes, Weiss voló a Pucallpa. Allí localizó un motor pero ningún bote, por lo que voló a Iquitos. Mientras Weiss viajaba por el río Ucayali en enero de 1961, el motor de su nuevo bote se descompuso, por lo que regresó a Iquitos, donde lo reparó y luego lo envió en barcaza a Pucallpa. Voló para encontrarse con el 
bote, no llegando de nuevo a Atalaya hasta marzo, después de una breve visita a Harner, que estaba en Imiríacocha en el río Tamaya. Nuevamente sufriendo problemas con el motor saliendo de Atalaya, Weiss voló a San Ramón y luego se fue en automóvil a Lima para hacer más reparaciones, llegando finalmente en abril a Atalaya, en avión.

El 4 de mayo de 1961, Weiss finalmente estaba en condiciones de buscar un sitio de campo en el río Tambo, dejando Atalaya con su equipo en dos botes - uno suyo, el otro perteneciente a un hombre llamado Trajano Izurieta- y en compañía de un guía, Carlos Vásquez. Después de una breve visita a la casa del patrón Carlos Ratteri, a quien se sintió obligado a visitar, Weiss se detuvo en la comunidad asháninka de Matobeni, donde se encontró con el profesor Santos, capacitado por el ILV. Continuando río arriba, en Anapati, Weiss conoció a un hombre asháninka llamado Luis Pérez, quien se ofreció a recibirlo, pero Weiss sintió que la comunidad estaba demasiado cerca de Atalaya. Pasó por Shoreni, donde radicaba Morán, otro maestro del ILV, pasando al asentamiento de un hombre llamado Gerónimo, luego a Santaro, luego de nuevo a Puerto Ocopa, donde fue recibido por la Madre Superiora, que "parecía un poco disgustada". E1 8 de mayo, Weiss regresó al asentamiento de Gerónimo, donde pasaría los siguientes meses. Las impresiones de Weiss allí, resumidas para el 10 a 12 de mayo, representan el detalle que caracteriza sus diarios personales y notas de campo.

La cabaña principal consiste en un techo sobre postes, bajo el cual hay 2 plataformas, donde los hombres (incluidos los visitantes) tienden a congregarse, recostados charlando o bebiendo masato. Debajo de las plataformas (una especialmente) se almacenan esteras, ollas, cestas. Desde el techo cuelgan varios tipos de cestas que contienen cosas, y adornos de plumas (llevados colgados atrás fuera del cushma [túnica]) y tambores. En el alero están colgados machetes, flautas de pan. Arriba se almacenan arcos y flechas y escopetas (Gerónimo tiene una que se carga por la boca del cañón), y remos. Depresiones en el suelo sostienen recipientes de masato, que se bebe con tazones de calabaza.

Las otras cabañas son lugares donde el trabajo de las mujeres tiende a realizarse: donde las hamacas de los bebés se cuelgan y mecen, donde se hila el hilo (el huso gira en un pequeño tazón de calabaza), donde se cocina la comida (la yuca es el alimento básico, aunque el maíz está colgada en la cabaña grande). El agua se trae del río en calabazas globulares con tapones de mazorca de maíz (ollas y utensilios de metal, y platos y cuencos esmaltados están incluidos). En la cabaña cerca de la cabaña grande y el camino al río se encuentra el comedero para elaborar masato. En la cabaña más lejana se está tejiendo una tela larga (de la que se cortaran un par de cushmas), la trama colgada de un árbol de papaya a unos 20 pies de distancia, con 2 soportes intermedios. Cestas (que se 
cargan a las espaldas colgadas de la frente) se utilizan para llevar las calabazas de agua y también para llevar la yuca traída de los campos. [...] Un par de pequeños gallineros con paredes están no muy lejos.

Estos meses tumultuosos dieron paso a un compromiso sostenido con el pueblo asháninka que duró muchos años. Gerald Weiss permaneció en la cuenca del Tambo desde mayo de 1961 hasta septiembre de 1962, después de lo cual pasó varios meses como becario Ogden Mills en el Museo Americano de Historia Natural. Estuvo allí nuevamente de julio de 1963 a agosto de 1964, cambiando de sitio de campo para vivir en Osherato con la familia de Matías Antúnez Gómez, con quien desarrollaría una amistad de por vida.

En este momento, no había documentación académica significativa del modo de vida asháninka. En este contexto, el objetivo de Weiss era "investigar todos los aspectos de la cultura [...] recopilando los datos necesarios para una descripción completa y precisa de esa cultura en su totalidad" (Weiss 1969:4). Como consecuencia, el trabajo de Weiss es increíblemente amplio y, como tal, es de gran valor para las generaciones actuales y futuras. Abarca no solo su investigación doctoral sobre cosmología, sino también la historia regional, la genealogía, numerosos aspectos de la cultura material (por ejemplo, manufacturas, subsistencia), el parentesco, el lenguaje y una colección de especímenes para la investigación etnobiológica, junto con objetos de cultura material.

Si bien el registro de publicaciones de Weiss relacionado con la cultura Asháninka es modesto (véase Weiss 1972, 1973, 1974, 1975, 1980, 1983, 1985, 2005), sus materiales inéditos son extensos, incluyendo manuscritos, diarios, notas, hojas fichas, correspondencia, grabaciones de historias y canciones y miles de fotografías. ${ }^{1}$ Los abundantes diarios y notas reflejan la actividad rica (y a veces onerosa) de la vida diaria, y las creencias y prácticas que la impregnan.

En particular, las contribuciones de Weiss a la documentación de la lengua asháninka son únicas. Por ejemplo, sus colaboraciones con biólogos y botánicos desde el Laboratorio de Entomología Sistemática hasta el Museo Field han dado como resultado un grado de precisión en la documentación léxica de Asháninka que es inusual en la documentación de las lenguas indígena (ver Figura 2, que también ilustra su documentación de las funciones de las plantas, y la variación dialectal).

1 Los materiales en papel, las grabaciones y las fotografías se archivarán en el California Language Archive (Universidad de California, Berkeley). Los bienes culturales materiales serán repatriados al Museo Nacional de la Cultura Peruana. 


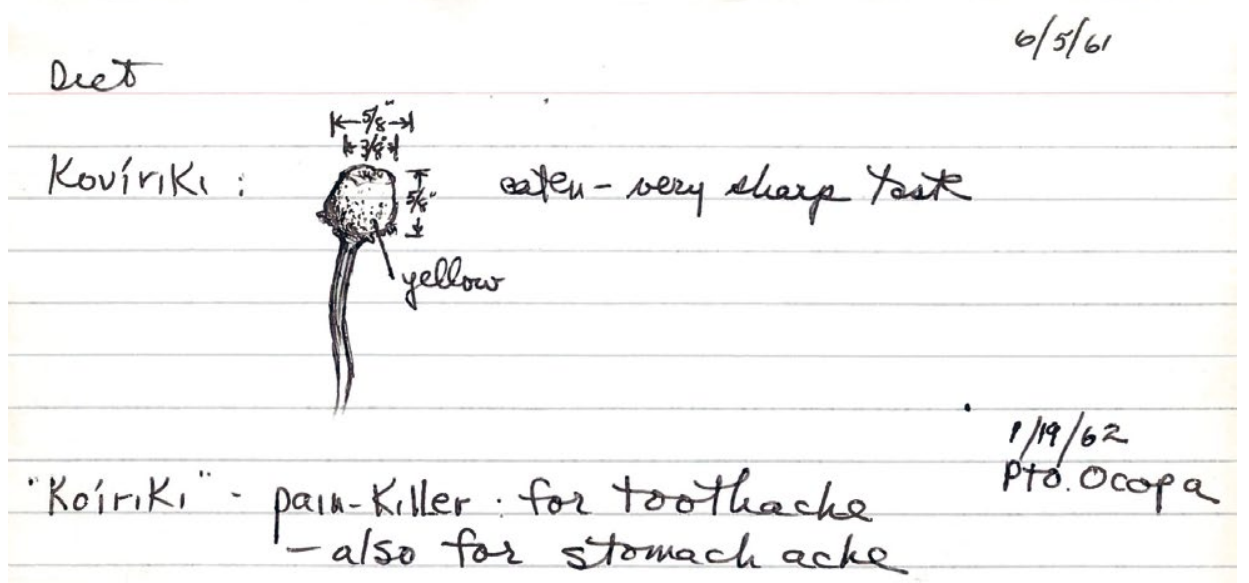

Figura 2: Ficha documentando kobiriki (Acmella oleácea), 5 de junio, 1961 (cortesía del California Language Archive).

Kovíriki: fruta amarilla, comestible, sabor muy picante

Koíriki-alivia dolor: para dolor de muela -también para dolor de estómago

En 1964, Weiss tomó un puesto como profesor de antropología en la Florida Atlantic University, donde permaneció hasta su jubilación en 2002. Durante este tiempo estuvo activo en asuntos sindicales, siendo vicepresidente y presidente del capítulo local de la United Faculty of Florida. En septiembre de 1976, Weiss recibió una beca que lo liberó de sus deberes de enseñanza, lo que le permitió montar una exposición de objetos de cultura material asháninka en la universidad (ver Riordan 1976), un ejemplo de la incorporación regular de las culturas indígenas sudamericanas en su enseñanza. El espíritu detrás de su trabajo etnográfico es evidente en una entrevista sobre la exposición (Harakas 1976).

Nosotros (los occidentales) siempre hemos tenido un fuerte impulso para imponer nuestra forma de vida a otras personas [...] ¿Por qué debemos forzar todo en un solo molde? ¿Por qué debemos homogeneizar toda la existencia?

Con su entonces esposa Sara y su hija Ana (y a veces solo), Weiss regresó a la cuenca del Tambo de septiembre a noviembre de 1977, de julio a diciembre de 1978 y de septiembre a noviembre de 1980, viviendo de nuevo con la familia 
de Matías Antúnez en la recién fundada comunidad de Otika. Después del viaje de 1977, estaba tan alarmado por los cambios que observó de los años transcurridos que diseñó e impartió un curso para el período de primavera de 1978 titulado "American Destiny". El espíritu detrás del curso es igualmente evidente en una entrevista (Sonsky 1978; véase también Weiss 1977).

La impresión que uno tiene ahora es que la historia se está repitiendo en América del Sur, la misma historia que ocurrió en América del Norte hace un siglo... Está ocurriendo allí justo ahora con la apertura por parte del gobierno de la mayor parte del interior de la cuenca amazónica. El resultado es que encontramos a estas tribus indias en peligro.

Después de 1980, las actividades de Sendero Luminoso hicieron inviable que Weiss regresara regularmente a la cuenca del Tambo. De hecho, en una carta escrita desde Sheboja el 22 de abril de 1991, Matías Antúnez dice "todos estamos en esta comunidad emos benido como refujido emos salido de Otica porque ha entrado los senderos [sic]". Weiss no regresó hasta 2009, acompañado por su hijo Eric.

El trabajo de Gerald Weiss está incrustado en un período temprano de investigación antropológica llevado a cabo en las comunidades Asháninka de la Selva Central y regiones adyacentes, junto con el de John Bodley, cuyo trabajo de campo comenzó en 1968 (Bodley 1970:v), John Elick (1919-2000), iniciado en 1951 (Elick 1969: xiii), William Denevan y Stefano Varese, quien, en marzo de 1964, apagando su motor se dirigió por el río Tambo y escuchando los Conciertos de Brandenburgo de Bach en la distancia, se encontró con el "amable y brillante" Weiss, "[un] joven delgado [que] lo recibió con la dignidad de alguien que ha vivido muchos meses aislado de su propia cultura y en el espacio liminal de otra que apenas está comenzando a revelarse" (Varese 2020:88). Este período, como se ha ilustrado anteriormente, fue uno en el que los proyectos de investigación de este tipo eran considerablemente más difíciles que otros similares en la actualidad. Sin embargo, el enfoque de Weiss encarna una amplitud de erudición que era inusual entonces y es aún más inusual hoy en día. Notablemente para la época, perfiló a sus maestros Asháninka en la presentación de sus observaciones de su modo de vida (Weiss 1969:10-14): Sabarojani, su esposa, Jebayoki, sus padres adoptivos, Shariti y Komempe, y el medio hermano de Sabarojani Kenchori, Porekabanti, Shirikonka, Komitya y Mariti. Este obituario está dedicado a ellos.

A Gerald Weiss le sobreviven su hija Ana y su hijo Eric. 


\section{Referencias bibliográficas}

Bodley, John. Campa Socio-economic Adaptation. PhD dissertation, University of Oregon: 1970.

Elick, John. An Ethnography of the Pichis Valley Campa of Eastern Peru. PhD dissertation, University of California, Los Angeles: 1969.

Harakas, Margo. "Living Among Andes Tribe Professor Learns from Primitives". In: Fort Lauderdale News, October 24, 1976, p. 142.

Riordan, Patrick. He Knows Why Peru's Indians Rub Sticks, Yet Carry Matches. The Miami Herald, October 16, 1976, p. 121.

Sonsky, Steve. "FAU Prof: Indians Threatened Again”. In: The Miami Herald, February 27, 1978, p. 110.

Varese, Stefano. The Art of Memory: An Ethnographer's Journey. Raleigh: Editorial A Contracorriente, 2020.

Weiss, Gerald. The Cosmology of the Campa Indians of Eastern Peru. 1969.

Weiss, Gerald. Campa Cosmology. Ethnology 11(2) (1972): 157-172.

Weiss, Gerald. A Scientific Concept of Culture. In: American Anthropologist 75 (1973): 1376-1413.

Weiss, Gerald. Campa Organization. In: American Ethnologist 1(2) (1974): 379-403.

Weiss, Gerald. Campa Cosmology: The world of a Forest Tribe in South America. AMNH. 1975.

Weiss, Gerald. "The Problem of Development in the Non-Western World". In: American Anthropologist 79(4) (1977): 887-893.

Weiss, Gerald. "The Aboriginal Culture Areas of South America”. In: Anthropos 75(3) (1980): 405-415.

Weiss, Gerald. “The Sun God and the Jaguars: A Campa Case of the Incomplete Assimilation of Myth". In: Latin American Indian Literatures 7(1) (1983): 1-12.

Weiss, Gerald. "Elements of Inkarrí East of the Andes”. In: Magaña, Edmundo \& Peter Mason (eds.), Myth and the Imaginary in the New World. 1985, pp. 305-320. 
Weiss, Gerald. Los campa ribereños. In: Santos-Granero, Fernando \& Frederica Barclay (eds.), Guía etnográfica de la Alta Amazonía. Institut Français d'Études Andines \& Smithsonian Tropical Research Institute, 2005, pp. 26-72.

Zachary O'Hagan

University of California, Berkeley (D) https://orcid.org/0000--0002-2720-2070 